
las Ciencias

ISSN: 1697-011X

revista.eureka@uca.es

Universidad de Cádiz

España

\title{
El banco de semillas del suelo. Una metodología experimental sencilla, reproducible y de bajo costo para aprender sobre la biología de las malezas
}

Foresto, Emiliano; Amuchástegui, María Andrea; Nuñez, Cesar Omar; Ibarra, Luis Exequiel

El banco de semillas del suelo. Una metodología experimental sencilla, reproducible y de bajo costo para aprender sobre la biología de las malezas

Revista Eureka sobre Enseñanza y Divulgación de las Ciencias, vol. 19, núm. 1, 2022

Universidad de Cádiz, España

Disponible en: https://www.redalyc.org/articulo.oa?id=92068491006

DOI: https://doi.org/10.25267/Rev_Eureka_ensen_divulg_cienc.2022.v19.11.1203 


\section{El banco de semillas del suelo. Una metodología experimental sencilla, reproducible y de bajo costo para aprender sobre la biología de las malezas}

The soil seed bank: A simple, reproducible and inexpensive experimental methodology to learn about the biology of weeds

Emiliano Foresto

Universidad Nacional de Rio Cuarto (UNRC), Rio

Cuarto, Córdoba., Argentina

eforesto@ayv.unrc.edu.ar

(iD) https://orcid.org/0000-0002-8196-3030

María Andrea Amuchástegui

Universidad Nacional de Rio Cuarto (UNRC), Rio

Cuarto, Córdoba., Argentina

aamuchastegui@ayv.unrc.edu.ar

(D) https://orcid.org/0000-0002-3682-6870

Cesar Omar Nuñez

Universidad Nacional de Rio Cuarto (UNRC), Rio

Cuarto, Córdoba., Argentina

cnunez@ayv.unrc.edu.ar

iD https://orcid.org/0000-0001-8744-3581

\section{Luis Exequiel Ibarra}

Universidad Nacional de Rio Cuarto (UNRC), Rio

Cuarto, Córdoba., Argentina

libarra@exa.unrc.edu.ar

(D) https://orcid.org/0000-0002-6077-7992
DOI: https://doi.org/10.25267/

Rev_Eureka_ensen_divulg_cienc.2022.v19.i1.1203

Redalyc: https://www.redalyc.org/articulo.oa?

$\mathrm{id}=92068491006$
Recepción: 24 Marzo 2021

Revisado: 19 Abril 2021

Aprobación: 07 Septiembre 2021

\section{Resumen:}

A pesar de la importancia que presentan las salidas al campo y el trabajo experimental en la sanidad vegetal, las carreras de Ingeniería Agronómica y similares carecen de estas actividades en sus programas. Para abordar esa deficiencia desarrollamos una propuesta basada en una clase experimental usando los bancos de semillas del suelo (BSS) para promover el estudio de la biología y ecología de las malezas en un curso de grado para aspirantes a Ingenieros Agrónomos. Durante el desarrollo de la propuesta, el alumnado realiza recolección de muestras del BSS en un campo agrícola-ganadero sometido a diferentes tipos de labranza y por medio de una metodología que combina salidas al campo, trabajo en laboratorio y procesamiento estadísticos de resultados, evaluando diferentes aspectos del BSS del área de estudio con la entrega de un informe final de la experiencia. La propuesta utiliza materiales de bajo costo y equipamiento que resulta de uso común en cualquier laboratorio universitario, la metodología es sencilla y fácilmente reproducible, como así también, libre de riesgos para la salud humana, siendo fácilmente aplicable en cualquier centro de enseñanza universitario o secundario. Este conjunto de experimentos debería ayudar al alumnado a desarrollar diferentes habilidades como el manejo y diseño de ensayos experimentales y a promover sus capacidades de discutir y resolver problemas prácticos, así como de sacar conclusiones de sus observaciones y los resultados obtenidos. Finalmente, esta actividad práctica les pone en contacto con su futura carrera profesional y, además, les brinda una posibilidad de iniciarse en el campo de la investigación. Esta propuesta tiene como objetivo aumentar la capacidad del alumnado para resolver problemas utilizando métodos científicos flexibles, desarrollar habilidades como el manejo de ensayos experimentales y la capacidad de discutir sus observaciones o resultados obtenidos. 
Palabras clave: banco de semilla del suelo, malezas, didáctica de las ciencias agronómicas, metodología experimental, educación superior.

\section{Abstract:}

Despite the importance of field trips and experimental works in plant health, Agronomic Engineering and similar careers lack these types of activities in their programs. To address this deficiency, we developed a proposal based on an experimental class using soil seed banks (SSB) to promote the study of the biology and ecology of weeds in an undergraduate course for aspiring agronomists. During the development of the proposal, the students collect samples from the SSB in an agricultural-livestock field subjected to different types of tillage and through a methodology that combines field trips, laboratory work and statistical processing of results, evaluating different aspects of the SSB of the study area with the delivery of a final report of the experience. The proposal uses low-cost materials and equipment that are commonly present in any university laboratory, the methodology is simple and easily reproducible, as well as free of risks to human health, being applicable in any university or secondary school. This set of experiments should help students to develop different skills such as the management and design of experimental trials, to promote their ability to discuss and solve practical problems, as well as to draw conclusions from their observations and the results obtained. Finally, this practical activity puts them in contact with their future professional career and also gives them a chance to start in the field of research. This proposal aims to increase the ability of students to solve problems using flexible scientific methods and develop skills such as handling experimental trials and discussing their observations or results obtained.

KEYWORDS: soil seed bank, weeds, didactics of agricultural sciences, experimental methodology, university education.

\section{INTRODUCCIÓN}

Una de las áreas más prometedoras de investigación de la biología y ecología de las malezas es la relacionada con los bancos de semillas del suelo (BSS). El estudio de la biología y ecología de las malezas como unidades temáticas abarcan un gran porcentaje del programa del curso de grado de Malezas en la carrera de Ingeniería Agronómica (IA) en cualquier universidad del mundo. En la asignatura se hace hincapié en el manejo integral de malezas con el objetivo de preparar al alumnado para predecir el impacto de las prácticas de manejo de las diferentes poblaciones de malezas a lo largo del tiempo. Se pone el foco en los ciclos de vida y crecimiento de las malezas, sus hábitos de crecimiento, sus estrategias reproductivas, la importancia del enmalezamiento en los sistemas de producción agrícola y el banco de semillas del suelo como un elemento central para la comprensión de la perpetuidad de las malezas en el medio ambiente. Definidos como una agregación de semillas no germinadas, los BSS son importantes en la ecología de las plantas porque tienen el potencial de reemplazar las plantas anuales adultas que mueren debido a que completan su ciclo de vida o alguna causa no natural, y las plantas perennes, que son susceptibles de morir por enfermedad, perturbación o consumo de animales (Leck, Parker y Simpson, 1989).

Existen varios métodos para estudiar los BSS, el más utilizado es el método de emergencia de las plántulas, que consiste en analizar la composición de las semillas en el suelo mediante la identificación de la nacencia de plántulas en un laboratorio o invernadero. Si bien se consigue mayor facilidad en la identificación de plántulas que de semillas, se puede subestimar el BSS si los requerimientos de germinación no son los adecuados. Otro método es el de identificación de las semillas tras su extracción por lavado y tamizado. En este método las semillas se separan del suelo y restos orgánicos a través de sucesivos lavados y tamizados con diferentes tamices que difieren en el tamaño de malla. La ventaja de este método es la reducción del volumen de la muestra de suelo, lo cual facilita la identificación posterior bajo el microscopio estereoscópico permitiendo encontrar semillas grandes y que puedan estar en estado latente (Acosta y Agüero, 2001)

La enseñanza de los conceptos de ecología vegetal en los cursos introductorios de biología a menudo se limita a lecturas de material teórico y conferencias magistrales a cargo del profesorado responsable debido a problemas logísticos con el trabajo de campo, y por lo tanto este tipo de actividades es una gran carencia en el aprendizaje de los estudiantes quienes llegan a cursos más avanzados con una aproximación muy alejada de la realidad del campo. Algunas limitantes se relacionan con el tiempo de viaje (distancia hasta el campo experimental), con un horario de clase programado que suele no ser suficiente para el análisis de la vegetación 
en el campo, lo que a menudo impide el estudio de las poblaciones y comunidades de plantas. Por otro lado, demasiados estudiantes en una clase (Simmons, 1998), tiempo limitado y recursos financieros escasos (Pascoe, 1994), falta de confianza sobre educación ecológica (Brewer, 2002) y otros desafíos representan limitaciones adicionales.

Una alternativa al estudio de la composición y dinámica de la comunidad de plantas in situ es recolectar muestras de suelo que contengan semillas enterradas (el banco de semillas), proceder a separar las semillas del suelo y los restos orgánicos, y finalmente, realizar la identificación de las especies vegetales para lograr entender la dinámica de la comunidad a través de la composición del BSS. Es importante trabajar con este método de muestreo ya que los BSS están compuestos de semillas enterradas latentes pero viables, las semillas pueden germinar inmediatamente, pero a menudo una proporción de las semillas producidas en una estación permanecerá enterrada en un estado latente y germinará de manera intermitente durante un largo período de tiempo. Las semillas corresponden a las especies que crecen actualmente en la superficie, a aquellas especies que existieron en el área en los últimos años, incluyendo así mismo semillas que han sido transportadas al área. Las semillas de cada especie tienen sus propios requisitos de germinación y solo germinaran cuando se cumplan unas condiciones específicas. Por lo tanto, las semillas pueden germinar y ser reclutadas en la comunidad cuando se cumplen las condiciones estacionales (Baskin y Baskin, 1985) o cuando una perturbación cree las condiciones adecuadas (Miles, 1979). El BSS también proporciona una fuente de novedad genética para especies de plantas. Por lo tanto, cada comunidad tiene un banco de semillas único que es un factor importante para determinar su composición florística (Amuchástegui, Nuñez, Balzola, Zorza y Mulko, 2012).

Debido a su simplicidad, el uso de bancos de semillas en el suelo puede proporcionar una método rápido y fácil para que el alumnado de grado y posgrado experimenten una situación de aproximación a lo que sucede en el campo y en el laboratorio. El trabajo de campo constituye por excelencia una estrategia pedagógica para la Educación Universitaria que permite integrar los contenidos disciplinares y científicos (Mayoral, 2019; Foresto y Martin, 2020).

Haciendo una exploración de la bibliografía existente que utiliza el BSS como recurso educativo encontramos algunos trabajos que refieren al BSS mediante la metodología de emergencia de plántulas en Educación Primaria (Ju y Kim, 2011; Ju y Kim, 2012) o Secundaria (Pascoe, 1994); mientras que solo un trabajo analizó el BSS en un curso de Educación Universitaria, utilizando el método de separación de semillas por flotación (Regnier, 1995). En este sentido, se advierte un vacio de investigaciones vinculadas al BSS en todos los niveles educativos, pero con mayor énfasis en Educación Superior.

El objetivo del trabajo se centra en el desarrollo de una actividad experimental que involucra el estudio del BSS de malezas en un campo agrícola como recurso didáctico. Mediante la utilización de procedimientos de campo y de laboratorio para su estudio, el alumnado tiene la posibilidad de aprender sobre la biología de las malezas. Este es un método que evita la destrucción significativa del hábitat, pero facilita al alumnado hacer observaciones cuidadosas de los propágulos de malezas que se encuentren en el suelo.

\section{OBJETIVOS DE APRENDIZAJE}

Tras finalizar la clase práctica, el alumnado puede:

- Objetivos metodológicos y experimentales:

- Caracterizar la composición y tamaño del BSS en los primeros $10 \mathrm{~cm}$ de profundidad de suelos sometidos a diferentes tratamientos de labranza.

- Comparar la composición de la vegetación establecida en el aérea con la composición del banco de semillas para determinar la contribución de los BSS a las comunidades de plantas sobre el suelo.

- Analizar la relación de la variable profundidad de suelo con la composición del BSS (en nuestro caso profundidades de entre $0-5$ y $5-10 \mathrm{~cm})$. 
- Visualizar el efecto de diferentes tratamientos de labranza en el banco de semillas.

- Adquirir a habilidades de campo vinculadas a la recolección de muestras de suelo y la identificación de especies vegetales en los censos de la vegetación establecida.

- Obtener entrenamiento en el laboratorio relacionadas con el procesamiento de muestras del suelo e identificación de semillas por medio de lupas estereoscópicas.

- Objetivos didácticos-educativos:

- Capacitarse en la interpretación, discusión y registro de resultados experimentales.

- Construir habilidades para trasmitir lo aprendido por medio del lenguaje escrito a través de la presentación de un informe de laboratorio.

- Construir habilidades para trasmitir lo aprendido por medio del lenguaje oral a través de la discusión de resultados y debate entre el alumnado.

- Construir habilidades vinculadas al pensamiento critico y el trabajo colaborativo con sus compañeros.

\section{Nivel de la actividad}

Esta propuesta didáctica con un carácter de actividad práctica fue implementada en un grupo de estudiantes que cursan su $4^{\circ}$ año del ciclo obligatorio, en la asignatura llamada Malezas, equivalente a Weed Science para países de habla inglesa. Participaron de la misma aproximadamente 60 alumnos. La asignatura pertenece al programa de la carrera de IA, pero la actividad también sería adecuada para otras ciencias agropecuarias o biológicas que indaguen en la biología y ecología de especies de plantas, como Licenciatura en Ciencias Biológicas, Profesorado en Biología, Ingeniería Forestal, entre otras. Además, realizando algunas adaptaciones según los objetivos que se persigan podría ser útil como una práctica transversal a los niveles de enseñanza de pregrado, grado y posgrado.

\section{Propuesta de implementación de la Clase}

\section{Clases de preparación y conocimientos previos requeridos por el alumnado.}

Antes de emprender esta actividad, el alumnado necesita recuperar conocimientos previos de biología, ecología, morfología vegetal, botánica y bioestadística, los cuales son adquiridos a través de cursos del plan de estudios del primer, segundo y tercer año de la mayoría de las carreras universitarias mencionadas anteriormente. En esta asignatura el objetivo educativo que se persigue es establecer relaciones causales entre la aparición de especies de malezas, sus propiedades biológicas y la influencia de factores abióticos y bióticos, principalmente los impactos antropogénicos a través de los diferentes métodos de manejo y control de malas hierbas en áreas destinadas a usos agrícolas-ganaderos.

También se requiere que el alumnado posea alguna experiencia con técnicas básicas de trabajo de campo y laboratorio como uso de lupas estereoscópicas, uso de estufas de secado y también, otras habilidades como la recopilación de datos con cuidado y precisión, conocimientos básicos de la aplicación del método científico.

\section{Esquema de la clase}

Para este trabajo experimental se ha propuesto que el alumnado se divida en grupos de 6 estudiantes para trabajar ya que según nuestra experiencia docente en los trabajos de campo y laboratorio esta cantidad de alumnos permite que todos puedan participar de las actividades y a su vez es un número de estudiantes en 
el que se facilita el trabajo en equipo, la comunicación y el intercambio de ideas durante el desarrollo de la propuesta.

El flujo de trabajo se organiza en tres clases consecutivas de 3 horas y en torno a diferentes momentos pedagógicos: apertura teórica, procedimientos experimentales (de campo y en laboratorio), discusión y evaluación (Tabla 1, Figura 1).

El flujo de trabajo experimental se detalla en la sección de metodología en el punto 4 de este escrito. Todos los procedimientos experimentales requieren el uso de poco equipamiento, ya que lo indispensable es contar con lupas estereoscópicas, una estufa de secado y heladera. Además de materiales como Erlenmeyer (remplazable por botellas de plástico de gaseosas o refrescos), cilindros metálicos para extracción de muestras, martillo, bolsas de nylon, cinta de papel y rotuladores. Las clases tienen riesgos mínimos para la salud. Al final, el alumnado comunica sus hallazgos en un informe escrito. 
TABLA 1

Diseño de clase práctica

\begin{tabular}{|c|c|c|c|}
\hline $\begin{array}{l}\text { Momentos } \\
\text { de la clase }\end{array}$ & Día & ¿En qué consiste cada momento? & ¿Qué se evaluará? \\
\hline $\begin{array}{l}\text { Apertura } \\
\text { teórica }\end{array}$ & $1^{\circ}$ & $\begin{array}{l}\text { Antes de la clase de campo, se ofrece un } \\
\text { seminario taller al alumnado mediante } \\
\text { una presentación de diapositivas en un } \\
\text { período de } 90 \text { minutos para discutir con- } \\
\text { ceptos relacionados a los diferentes tipos } \\
\text { de BSS, la extracción de muestras de este, } \\
\text { los métodos de separación del BSS, la di- } \\
\text { námica de la población de semillas en el } \\
\text { suelo, las formas de dispersión de las mis- } \\
\text { mas y formas de tabulación de los datos, } \\
\text { expresión e interpretación de los resulta- } \\
\text { dos. }\end{array}$ & $\begin{array}{l}\text {-Participación en el seminario mediante } \\
\text { las intervenciones, el debate, formula- } \\
\text { ción de preguntas que busque una } \\
\text { perspectiva de acuerdo y/o desacuerdo. } \\
\text { Este aspecto será evaluado mediante la } \\
\text { observación de los alumnos en el desa- } \\
\text { rrollo de la apertura teórica. }\end{array}$ \\
\hline $\begin{array}{l}\text { Metodología } \\
\text { experimental } \\
\text { (Campo - } \\
\text { Laboratorio } \\
\text { de semillas) }\end{array}$ & $\begin{array}{l}1^{\circ}, 2^{\circ} \\
\mathrm{y} 3^{\circ}\end{array}$ & $\begin{array}{l}\text {-Mediante la utilización de una guía de } \\
\text { trabajo práctica impresa el alumnado tra- } \\
\text { bajará elaborando un plan de acción deri- } \\
\text { vado de las bases y metodologías propor- } \\
\text { cionadas en la guía que son detalladas en } \\
\text { el punto } 4 \text { de este trabajo. } \\
\text {-Se pretende que todo el alumnado traba- } \\
\text { je en la experiencia, es decir pueda: tocar, } \\
\text { manipular, hacer, probar, etc. } \\
\text { Se pretende que alimenten el desarrollo } \\
\text { de actitudes críticas y pensamientos sobre } \\
\text { lo experimental realizado. }\end{array}$ & $\begin{array}{l}\text {-Participación en las tareas a campo y en } \\
\text { el trabajo en el laboratorio. } \\
\text {-Capacidad para manipular materiales de } \\
\text { laboratorio. } \\
\text {-Experticia para formar criterios y to- } \\
\text { mar decisiones. } \\
\text { Este aspecto será evaluado mediante la } \\
\text { observación del alumnado en el desa- } \\
\text { rrollo de la los trabajos experimentales. }\end{array}$ \\
\hline $\begin{array}{l}\text { Discusión de } \\
\text { resultados }\end{array}$ & $3^{\circ}$ & $\begin{array}{l}\text {-El proceso de inferencias y conclusiones } \\
\text { se comparte exponiendo las actividades } \\
\text { realizadas y los resultados obtenidos de } \\
\text { su trabajo experimental. } \\
\text { - Se realiza una discusión grupal, donde } \\
\text { se evidenciarán puntos de acuerdos, des- } \\
\text { acuerdos, unificación de criterios, búsque- } \\
\text { da de consensos y puntos comunes, } \\
\text { perspectivas y posibilidades de aplicación. } \\
\text {-El objetivo final es que el alumnado ad- } \\
\text { quiera los criterios que necesitarán duran- } \\
\text { te su vida profesional. }\end{array}$ & $\begin{array}{l}\text { Habilidad para la construcción de con- } \\
\text { ceptos y la comunicación de los mismo } \\
\text { de forma oral con sus pares. Este aspec- } \\
\text { to será evaluado mediante la observa- } \\
\text { ción del alumnado durante la discusión } \\
\text { de resultados. Aquí se observará la capa- } \\
\text { cidad de formar un criterio, de argu- } \\
\text { mentar y contrargumentar, discutir y } \\
\text { analizar los resultados obtenidos duran- } \\
\text { te sus trabajos experimentales. }\end{array}$ \\
\hline $\begin{array}{l}\text { Evaluación } \\
\text { de los apren- } \\
\text { dizajes }\end{array}$ & $\begin{array}{l}1^{\circ}, 2^{\circ} \\
\text { y } 3^{\circ}\end{array}$ & $\begin{array}{l}\text {-Esto pretende una evaluación holística } \\
\text { de todo el proceso de aprendizaje. En } \\
\text { este apartado se pensó solicitar al alum- } \\
\text { nado la elaboración de un informe escrito } \\
\text { que debe ser entregado transcurridos } 15 \\
\text { días desde la finalización de las activida- } \\
\text { des del } 3^{\circ} \text { día. El mismo debía ser devuel- } \\
\text { to al alumnado corregido } 7 \text { días posterio- } \\
\text { res a la entrega del informe. Para el infor- } \\
\text { me se fijaron algunos criterios que debían } \\
\text { ser conocidos con anterioridad por el } \\
\text { alumnado por medio de una rúbrica que } \\
\text { adaptada según Foresto et al. (2021), in- } \\
\text { cluida en la tabla } 2 \text {. }\end{array}$ & $\begin{array}{l}\text { Capacidad para comunicar lo aprendido } \\
\text { a través del lenguaje escrito por el infor- } \\
\text { me de reporte de actividades. }\end{array}$ \\
\hline
\end{tabular}




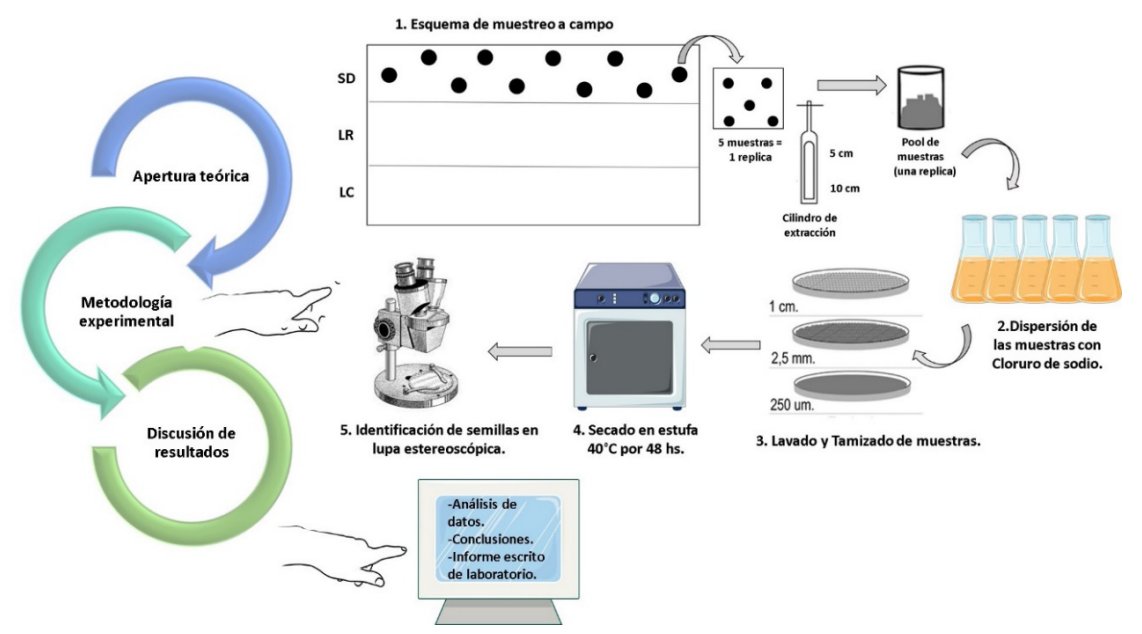

FIGURA 1

Flujo de trabajo de la clase con sus diferentes momentos. Esta figura muestra una representación esquemática del diseño de la clase. Los momentos de la clase están marcados con diferentes colores: azul es la apertura teórica de la actividad inicial apertura de la clase donde los instructores deben explicar los antecedentes teóricos en base a los conceptos descritos en la sección de introducción, verde agua es el procedimiento experimental en el que se esquematiza todo el procedimiento de trabajo y verde corresponde a la finalización de la clase donde se discuten los resultados.

\section{METOdología EXPERIMENTAL PARA LOS PROCEDIMIENTOS DE LABORATORIO Y DE CAMPO}

\section{Área de estudio}

El sitio experimental usado para este trabajo práctico fue un campo agrícola-ganadero de la provincia de Córdoba, Argentina. El suelo donde se realizó este trabajo es un haplustol típico, desarrollado sobre un material franco arenoso muy fino. En este sentido resulta interesante destacar que el área de estudio va a depender de los objetivos del curso. En nuestro caso al tratarse de una actividad destinada para Ingenieros Agrónomos se eligió un campo agrícola con presencia de malezas. El área de estudio podría involucrar estudio de pastizales naturales bajo diferentes tipos de laboreo (Leguizamón y Roberts 1982; Pucheta, Cabido, Díaz y Funes 1998), áreas que sufrieron disturbios como el fuego o el pastoreo (Ernst y Morici 2013) o el estudio de bosques nativos (Sione, Ledesma, Rosenberger, Galliussi y Sabattini 2015; Sabattini, Sione, Ledesma, Rosenberger, Wilson, Loker y Cinto 2018), entre otros.

\section{Diseño experimental}

El diseño experimental utilizado fue completamente aleatorizado. El BSS fue muestreado en marzo, luego de la diseminación de las especies estivales, y previo a la germinación de las malezas invernales.

Se trabajó sobre un ensayo de sistemas de labranzas con una rotación agrícola-ganadera. Estos sistemas fueron conducidos con tres sistemas de labranza:

- Siembra directa (SD): sin remoción del suelo.

- Labranza reducida (LR): se realizó la labranza vertical usando Para-till y rastra de discos de tiro excéntrico.

-Labranza convencional (LC): se realizó la labranza vertical en base a arado de cincel y rastra de discos de tiro excéntrico. 
Para cada tratamiento (SD, LR y LC) se tomaron 10 muestras compuestas de cinco submuestras de suelo. Para ello, se utilizó un cilindro de $3 \mathrm{~cm}$ de diámetro por $10 \mathrm{~cm}$ de longitud, separando en diferentes profundidades $(0-5 \mathrm{~cm}$ y $5-10 \mathrm{~cm})$. Cada profundidad fue muestreada en forma independiente. El tamaño total del BSS fue considerado de $0 \mathrm{a} 10 \mathrm{~cm}$ de profundidad. El alumnado caminó cada parcela de tratamiento en Forma de Mo W para tomar muestras en diferentes puntos del lote.

\section{Procesamiento de las muestras}

El volumen de suelo extraído por cada tratamiento fue de $0,0010 \mathrm{~m}^{3}$, siendo equivalente a una superficie de suelo de $0,0106 \mathrm{~m}^{2}$. La expresión de los resultados en metro cuadrado fue preferida para mejorar la comprensión y además facilitar la comparación con otros trabajos. Para el análisis de las réplicas se utilizó el método de extracción por lavado y tamizado para ello las réplicas de suelo correspondientes a cada tratamiento fueron secadas a temperatura ambiente, almacenadas en un lugar oscuro y fresco hasta su procesamiento. A su turno, fueron disgregadas y pasadas por un tamiz de $5 \mathrm{~mm}$ de malla a fin de eliminar los restos orgánicos y pedregullos. Posteriormente las réplicas fueron homogeneizadas para sacar una alícuota de 500 gr destinada a la extracción de semillas. Cada alícuota de suelo se colocó en una botella de plástico de 2,5 L, agregándole $0,075 \mathrm{~kg}$ de sal y agua hasta llenar la misma. Para proceder a la etapa de dispersión de las muestras de suelo. De esta manera las botellas se agitaron fuertemente en forma manual y se dejaron reposar 48 hs. Luego se filtró el contenido a través de dos tamices con malla de $2 \mathrm{~mm}$ y 0,25 mm, respectivamente. Se lavó con agua varias veces para separar el suelo de las semillas, el producto del filtrado se colocó en papel de diario, se rotuló y se secó en estufa a $40^{\circ} \mathrm{C}$, durante 48 hs (Foresto, Amuchastegui, Nuñez, Zorza, 2021). Finalmente se procedió a la identificación y cuantificación de las semillas colocadas en una caja de Petri y con un microscopio estereoscópico, utilizando claves taxonómicas y bibliografía específica, siendo el alumnado el encargado del trabajo de laboratorio (figura 1).

\section{Análisis estadístico}

Los datos se analizaron siguiendo el formato de Foresto et al. (2021) a través de un ANAVA de una o dos vías de acuerdo al caso, con comparación de medias con test Tukey o Prueba de Kruskal-Wallis con test de rangos. Se analizó la riqueza, y la diversidad específica a través del índice de Shannon-Weaver. El análisis estadístico se ejecutó con el programa InfoStat (Di rienzo, Casanoves, Balzarini, Gonzalez,Tablada y Robledo, 2018). Para los test estadísticos se utilizó el nivel de significancia de $(p<0,05)$.

\section{Instrucciones de manipulación de muestras del banco de semillas}

En este apartado brindamos algunas recomendaciones necesarias para que los estudiantes tengan en cuenta a la hora de manipular muestras de BSS producto de nuestra experiencia con la temática:

- El tamaño mínimo de muestra debe ser de $500 \mathrm{~g}$.

- Se debe guardar en un recipiente a prueba de humedad, como una bolsa Ziploc de peso pesado o un recipiente de plástico con tapa hermética. Doble bolsa si la muestra contiene material afilado que puede perforar una bolsa (como restos orgánicos de ramas, raíces, etc.)

- La muestra puede estar seca, húmeda o mojada según las condiciones del campo. Si se moja, la muestra debe ser procesada lo antes posible para reducir el riesgo de que las semillas rompan la dormición y germinen durante el transporte. 
- Si la muestra está contaminada con una sustancia química conocida, tenga cuidado durante el trabajo de campo (por ejemplo, algún agroquímico o fertilizante)

- La muestra debe ser procesada lo antes posible después del muestreo y manteniéndola fresca (en el refrigerador a $4^{\circ} \mathrm{C}$ ) y oscura para retardar la germinación de las semillas.

- Es importante incluir la siguiente información: nombre del grupo de estudiantes, número de lote u otra información de identificación, fecha de muestreo, información sobre el tipo de labranza, cualquier otra instrucción especial que sea valiosa a la hora del análisis de los datos.

\section{RESULTADOS Y Discusión}

Esta clase experimental se organiza en cuatro momentos pedagógicos: actividad teórica, procedimientos experimentales llevados a cabo a campo y en el laboratorio de semillas, discusión y evaluación (Tabla 1). A su vez estos momentos se distribuyen diferencialmente durante tres clases de laboratorio

\section{Día 1 (primera semana)}

El primer día, el profesorado encargado de las actividades prácticas explica las bases conceptuales teóricas y la relevancia del estudio a realizar, los objetivos y la organización de los procedimientos experimentales. Se invita al alumnado a realizar una lluvia de ideas sobre la temática y deberán plasmarla en la pizarra. Posteriormente el profesorado, agregara conceptos de importancia que puedan faltar, pidiendo al alumnado que analice la lógica subyacente a los objetivos establecidos y le ayuda a pensar en cómo las tareas propuestas, imitan a las que se les pedirá que realicen a diario como parte de su trabajo profesional. De esta manera, se induce a al alumnado a activar sus conocimientos o aprendizajes informales relacionados con el tema en cuestión, que serán recuperados en este contexto formal de aprendizaje (Foresto, 2020).

Después de la introducción teórica, la segunda mitad de la clase continua con los procedimientos experimentales diseñados para esta actividad. El alumnado es llevado al campo agrícola-ganadero y se les presenta el diseño experimental planteado donde se tomarán las muestras (ver puntos 4.1, 4.2 y 4.5). El alumnado en grupos realiza un recorrido en los lotes y toma datos que pueda ser de su interés. En este caso deben realizar el relevamiento florístico de la vegetación establecida porque este dato se vuelve relevante a la hora de analizar las especies que se encuentran en el BSS. Luego de esto, el alumnado recorre el lote y junta muestras de los diferentes tratamientos las cuales son guardadas en bolsas de nylon. Si bien el trabajo de toma de muestras puede parecer algo automatizado se debe prestar mucha atención: a) en enterrar los cilindros en su totalidad para abarcar los $10 \mathrm{~cm}$ de profundidad, b) a la hora de retirar las muestras de suelo del cilindro debe asegurarse de retirar todo el contenido. Estas dos consideraciones son importantes para evitar errores de muestreo.

Para facilitar la toma de muestras que es un trabajo arduo, cada grupo se hará cargo de la toma de 20 muestras (10 muestras de cada profundidad) y se les asignará una determinada labranza. De esta manera el alumnado durante los días posteriores trabajara en el procesamiento de las muestras, y para la confección del informe final podrán intercambiar datos con compañeros y cada grupo analizara por completo los tres tratamientos en las dos profundidades.

Una vez que el alumnado junta todas sus muestras, debe exponer las muestras en el laboratorio para su secado a temperatura ambiente y luego realizar la separación de los restos orgánicos (trozos de raíces, tallos etc.). Ya con las muestras reducidas se procede a realizar la dispersión de las mismas con cloruro de sodio (ver punto 4.3) en los Erlenmeyer o botellas de plástico durante $48 \mathrm{hs}$. Si por los requerimientos de la clase este punto no puede realizarse de manera inmediata como se plantea, las muestras pueden ser llevadas a la 
heladera $\left(4^{\circ} \mathrm{C}\right)$ para evitar la germinación de algunas semillas que durante este trabajo puedan romper su dormición (figura 2).

\section{Día 2 (segunda semana)}

El segundo día, el alumnado continúa con los procedimientos experimentales en los laboratorios. Aquí se vuelcan las muestras que están en las botellas sobre los tamices y se hace correr agua ayudándose con sus manos hasta quitar todos los excedentes de tierra. La idea es que en los tamices solo queden los propágulos. El alumnado coloca las muestras lavadas y tamizadas en un papel de diario o sobre de papel madera el cual es rotulado con marcadores y luego son llevados a las estufas de secado por lo menos 48 horas y así las muestras quedan listas para pasar a la siguiente etapa (ver punto 4.3).

Si bien el trabajo en ese día es bastante repetitivo, es una tarea que lleva tiempo por lo que el alumnado destina dos horas del práctico al proceso del lavado y tamizado, durante la hora restante se abordarán dudas sobre el procedimiento experimental, como así también explicarán pautas para la realización del informe final y los parámetros cuali-cuantitativos que se tendrán en cuenta para su evaluación (ver tabla 2). 
TABLA 2

Rúbrica de calificación cuali-cuantitativa del informe del laboratorio del BSS

\begin{tabular}{|c|c|c|c|c|}
\hline & \multicolumn{4}{|c|}{ Criterios de evaluación (cualitativos y cuantitativos) } \\
\hline $\begin{array}{l}\text { Introducción } \\
\text { y marco teóri- } \\
\text { co } \\
\text { (máx. } 16 \text { pun- } \\
\text { tos) }\end{array}$ & $\begin{array}{l}\text { - Se proporciona } \\
\text { una descripción } \\
\text { completa del esta- } \\
\text { do del arte de la } \\
\text { temática. } \\
\text {-El alumnado ex- } \\
\text { plica adecuada- } \\
\text { mente la impor- } \\
\text { tancia del estudio } \\
\text { y por qué es im- } \\
\text { portante realizar } \\
\text { estos ensayos. } \\
\text {-El alumnado des- } \\
\text { cribe en detalle las } \\
\text { aplicaciones ac- } \\
\text { tuales y la impor- } \\
\text { tancia de los BSS. } \\
\text { 14-16 puntos }\end{array}$ & $\begin{array}{l}\text { - Se proporciona al- } \\
\text { guna descripción del } \\
\text { estado del arte de la } \\
\text { temática. } \\
\text {-El alumnado explica } \\
\text { sin mayores detalles } \\
\text { la importancia del es- } \\
\text { tudio y por qué es } \\
\text { importante realizar } \\
\text { estos ensayos. } \\
\text { - El alumnado descri- } \\
\text { be algunas de las } \\
\text { aplicaciones actuales } \\
\text { y la importancia de } \\
\text { los BSS. } \\
\text { 10-12 puntos }\end{array}$ & $\begin{array}{l}\text { - Se proporciona una } \\
\text { vaga descripción del } \\
\text { estado del arte de la } \\
\text { temática. } \\
\text {-El alumnado ofrece } \\
\text { una pobre descripción } \\
\text { de la importancia del } \\
\text { estudio y por qué es } \\
\text { importante realizar es- } \\
\text { tos ensayos. } \\
\text { - El alumnado descri- } \\
\text { be pocas de las aplica- } \\
\text { ciones actuales e im- } \\
\text { portancias de los BSS. } \\
\text { 6-8 puntos }\end{array}$ & $\begin{array}{l}\text { - El estudiante no } \\
\text { proporciona ninguna } \\
\text { descripción del esta- } \\
\text { do del arte de la te- } \\
\text { mática. } \\
\text {-El alumnado ofrece } \\
\text { una muy pobre o } \\
\text { ninguna descripción } \\
\text { de la importancia del } \\
\text { estudio y por qué es } \\
\text { importante realizar } \\
\text { estos ensayos. } \\
\text { - El alumnado des- } \\
\text { cribe muy pocas o } \\
\text { ninguna de las apli- } \\
\text { caciones actuales e } \\
\text { importancias de los } \\
\text { BSS. } \\
\text { 0-5 puntos }\end{array}$ \\
\hline $\begin{array}{l}\text { Materiales y } \\
\text { Métodos } \\
\text { (máx. } 20 \text { pun- } \\
\text { tos) }\end{array}$ & $\begin{array}{l}\text {-El alumnado ex- } \\
\text { plica claramente } \\
\text { cómo se utilizó la } \\
\text { metodología ade- } \\
\text { cuada para realizar } \\
\text { los diferentes en- } \\
\text { sayos. Además, los } \\
\text { materiales y méto- } \\
\text { dos son correctos } \\
\text { y están bien orga- } \\
\text { nizados. } \\
16-20 \text { puntos }\end{array}$ & $\begin{array}{l}\text {-El alumnado pro- } \\
\text { porciona una explica- } \\
\text { ción sobre cómo se } \\
\text { utilizó la metodolo- } \\
\text { gía adecuada para } \\
\text { realizar los diferentes } \\
\text { ensayos. Los materia- } \\
\text { les y métodos contie- } \\
\text { nen errores menores } \\
\text { y / o están algo de- } \\
\text { sorganizados. } \\
10-14 \text { puntos }\end{array}$ & $\begin{array}{l}\text {-El alumnado propor- } \\
\text { ciona información } \\
\text { inadecuada sobre } \\
\text { cómo se utilizó la me- } \\
\text { todología adecuada } \\
\text { para realizar los dife- } \\
\text { rentes ensayos. Los } \\
\text { materiales y métodos } \\
\text { contienen varios erro- } \\
\text { res y / o son difíciles } \\
\text { de interpretar. } \\
\mathbf{5 - 9} \text { puntos }\end{array}$ & $\begin{array}{l}\text { - El alumnado pro- } \\
\text { porciona una expli- } \\
\text { cación deficiente o } \\
\text { nula sobre cómo se } \\
\text { utilizó la metodolo- } \\
\text { gía adecuada para } \\
\text { realizar los diferentes } \\
\text { ensayos. Los mate- } \\
\text { riales y métodos } \\
\text { contienen múltiples } \\
\text { errores y /o son im- } \\
\text { posibles de interpre- } \\
\text { tar. } \\
\text { 0-4 puntos }\end{array}$ \\
\hline $\begin{array}{l}\text { Resultados y } \\
\text { discusión } \\
\text { (máx. } 20 \text { pun- } \\
\text { tos) }\end{array}$ & $\begin{array}{l}\text {-Todos los resul- } \\
\text { tados se explican } \\
\text { correctamente. } \\
\text {-Se incluyen foto- } \\
\text { grafías o figuras } \\
\text { de los resultados } \\
\text { obtenidos con su } \\
\text { leyenda explicati- } \\
\text { va. } \\
\text {-El alumnado rea- } \\
\text { liza una discusión } \\
\text { de los resultados } \\
\text { basándose en an- } \\
\text { tecedentes biblio- } \\
\text { gráficos consulta- } \\
\text { dos. } \\
\text { 16-20 puntos }\end{array}$ & $\begin{array}{l}\text {-Algunos de los re- } \\
\text { sultados se explican } \\
\text { correctamente. } \\
\text {-Se incluyen algunas } \\
\text { fotografías o figuras } \\
\text { de los resultados ob- } \\
\text { tenidos con su leyen- } \\
\text { da explicativa. } \\
\text {-El alumnado realiza } \\
\text { una discusión de al- } \\
\text { gunos resultados ba- } \\
\text { sándose en antece- } \\
\text { dentes bibliográficos } \\
\text { consultados. } \\
\text { 10-14 puntos }\end{array}$ & $\begin{array}{l}\text {-Muy pocos resultados } \\
\text { se explican correcta- } \\
\text { mente, la mayoría son } \\
\text { inconsistentes } \\
\text {-Se incluyen pocas fo- } \\
\text { tografías o figuras de } \\
\text { los resultados obteni- } \\
\text { dos y su leyenda es } \\
\text { inadecuada. } \\
\text {-El alumnado realiza } \\
\text { pocas discusiones de } \\
\text { los resultados y en la } \\
\text { mayoría de los casos } \\
\text { no se apoyan en ante- } \\
\text { cedentes bibliográfi- } \\
\text { cos } \\
\text { 5-9 puntos }\end{array}$ & $\begin{array}{l}\text {-Los resultados no se } \\
\text { explican correcta- } \\
\text { mente. } \\
\text {-Se incluyen muy po- } \\
\text { cas o ninguna foto- } \\
\text { grafías o figuras de } \\
\text { los resultados obte- } \\
\text { nidos con su leyenda } \\
\text { explicativa. } \\
\text {-El alumnado realiza } \\
\text { una discusión de los } \\
\text { resultados basándose } \\
\text { en antecedentes bi- } \\
\text { bliográficos consul- } \\
\text { tados o la discusión } \\
\text { es muy pobre. } \\
0-4 \text { puntos }\end{array}$ \\
\hline
\end{tabular}


TABLA 2

Continuación

\begin{tabular}{|c|c|c|c|c|}
\hline & \multicolumn{4}{|c|}{ Criterios de evaluación (cualitativos y cuantitativos) } \\
\hline $\begin{array}{l}\text { Conclusión o } \\
\text { consideracio- } \\
\text { nes finales y } \\
\text { perspectivas } \\
\text { futuras } \\
\text { (máx. } 20 \\
\text { puntos) }\end{array}$ & $\begin{array}{l}\text {-Se proporciona una ex- } \\
\text { plicación apropiada } \\
\text { para cualquier resultado } \\
\text { incorrecto o inconsis- } \\
\text { tente. } \\
\text {-Se proporciona un re- } \\
\text { sumen apropiado de los } \\
\text { resultados determina- } \\
\text { dos por sus experimen- } \\
\text { tos. } \\
\text {-El alumnado propor- } \\
\text { ciona información de } \\
\text { antecedentes adecuada } \\
\text { relacionada con el estu- } \\
\text { dio de fuentes externas } \\
\text { (texto, recursos web, ar- } \\
\text { tículos científicos). } \\
\text { - Se proporcionan posi- } \\
\text { bles perspectivas futu- } \\
\text { ras de investigación. } \\
16-20 \text { puntos }\end{array}$ & $\begin{array}{l}\text {-Se proporciona al- } \\
\text { guna explicación } \\
\text { para cualquier resul- } \\
\text { tado incorrecto o in- } \\
\text { consistente. } \\
\text {-Se proporciona al- } \\
\text { gún resumen de los } \\
\text { resultados determi- } \\
\text { nados por sus expe- } \\
\text { rimentos. } \\
\text { - El alumnado pro- } \\
\text { porciona alguna in- } \\
\text { formación básica re- } \\
\text { lacionada con el es- } \\
\text { tudio de fuentes ex- } \\
\text { ternas (texto, recur- } \\
\text { sos web, artículos } \\
\text { científicos). } \\
\text {-Se proporcionan al- } \\
\text { gunas posibles } \\
\text { perspectivas futuras } \\
\text { de investigación. } \\
10-14 \text { puntos }\end{array}$ & $\begin{array}{l}\text {-Se proporciona una } \\
\text { pequeña explicación } \\
\text { de los resultados in- } \\
\text { correctos o incon- } \\
\text { sistentes } \\
\text {-Se proporciona un } \\
\text { pequeño resumen } \\
\text { de los resultados de- } \\
\text { terminados por sus } \\
\text { experimentos } \\
\text { - El alumnado pro- } \\
\text { porciona poca o ine- } \\
\text { xacta información } \\
\text { de antecedentes re- } \\
\text { lacionada con el es- } \\
\text { tudio de fuentes ex- } \\
\text { ternas (texto, recur- } \\
\text { sos web, artículos } \\
\text { científicos). } \\
\text {-Se proporcionan } \\
\text { pocas perspectivas } \\
\text { futuras posibles de } \\
\text { investigación. } \\
5-9 \text { puntos }\end{array}$ & $\begin{array}{l}\text {-No se proporciona } \\
\text { ninguna explicación } \\
\text { de los resultados inco- } \\
\text { rrectos o inconsisten- } \\
\text { tes. } \\
\text {-No se proporciona } \\
\text { ningún resumen de } \\
\text { los resultados obteni- } \\
\text { dos en sus experimen- } \\
\text { tos. } \\
\text { - El alumnado no pro- } \\
\text { porciona antecedentes } \\
\text { relacionados con el } \\
\text { estudio de fuentes ex- } \\
\text { ternas (texto, recursos } \\
\text { web, artículos científi- } \\
\text { cos). } \\
\text { - No se proporcionan } \\
\text { posibles perspectivas } \\
\text { futuras de investiga- } \\
\text { ción. } \\
\text { 0-4 puntos }\end{array}$ \\
\hline $\begin{array}{l}\text { Referencias } \\
\text { bibliográficas } \\
\text { (máx. } 12 \\
\text { puntos) }\end{array}$ & $\begin{array}{l}\text {-Las referencias y citas } \\
\text { se utilizan correctamen- } \\
\text { te } \\
\text {-Todas las referencias } \\
\text { utilizadas son apropia- } \\
\text { das } \\
\text { - se respeta el formato } \\
\text { de referencia APA. } \\
\mathbf{1 0 - 1 2} \text { puntos }\end{array}$ & $\begin{array}{l}\text {-Las referencias y ci- } \\
\text { tas a veces se utilizan } \\
\text { correctamente } \\
\text {-La mayoría de las } \\
\text { referencias son apro- } \\
\text { piadas. } \\
\text {-Se respeta el forma- } \\
\text { to de referencia } \\
\text { APA. } \\
\text { 6-9 puntos }\end{array}$ & $\begin{array}{l}\text {-Faltan referencias o } \\
\text { citas o no se utilizan } \\
\text { correctamente } \\
\text {-Algunas referencias } \\
\text { son apropiadas. } \\
\text { - El formato de re- } \\
\text { ferencia APA es } \\
\text { poco respetado. } \\
\text { 1-5 puntos }\end{array}$ & $\begin{array}{l}\text { Faltan referencias y ci- } \\
\text { tas o no se utilizan co- } \\
\text { rrectamente. } \\
\text {-Las referencias no } \\
\text { son apropiadas. } \\
\text { - El formato de refe- } \\
\text { rencia APA es muy } \\
\text { poco respetado o no } \\
\text { se respeta. } \\
\mathbf{0} \text { puntos }\end{array}$ \\
\hline $\begin{array}{l}\text { Estructura y } \\
\text { formato gene- } \\
\text { ral del docu- } \\
\text { mento } \\
\text { (máx. } 12 \\
\text { points) }\end{array}$ & $\begin{array}{l}\text {-Todos los temas se tra- } \\
\text { tan en las secciones co- } \\
\text { rrespondientes. } \\
\text {-El documento está li- } \\
\text { bre de errores ortográ- } \\
\text { ficos y gramaticales. } \\
\text {-El texto sigue un orden } \\
\text { lógico y es fácil de en- } \\
\text { tender } \\
\text {-Se utilizan una fuente y } \\
\text { un diseño de página } \\
\text { adecuados. } \\
\text {-Se proporciona un títu- } \\
\text { lo apropiado. } \\
\text { - Se incluye una carátula } \\
\text { de presentación (con el } \\
\text { nombre, el instructor y } \\
\text { la sección de su } \\
\text { clase).10-12 puntos }\end{array}$ & $\begin{array}{l}\text {-La mayoría de los } \\
\text { temas se tratan en las } \\
\text { secciones correspon- } \\
\text { dientes. } \\
\text { - El documento con- } \\
\text { tiene pocos errores } \\
\text { ortográficos y / o } \\
\text { gramaticales. } \\
\text {-El texto es bastante } \\
\text { fácil de seguir y com- } \\
\text { prender. } \\
\text {-La fuente y el dise- } \\
\text { ño de la página son } \\
\text { aceptables. } \\
\text { 6-9 puntos }\end{array}$ & $\begin{array}{l}\text {-Algunos temas se } \\
\text { tratan en las seccio- } \\
\text { nes correspondien- } \\
\text { tes. } \\
\text {-El documento con- } \\
\text { tiene algunos erro- } \\
\text { res ortográficos y / } \\
\text { o gramaticales } \\
\text {-Algunos textos no } \\
\text { siguen un orden ló- } \\
\text { gico y son difíciles } \\
\text { de entender } \\
\text {-La fuente y diseño } \\
\text { de página general- } \\
\text { mente aceptables. } \\
\text { 1-5 puntos }\end{array}$ & $\begin{array}{l}\text {-Muchos temas están } \\
\text { ubicados en las sec- } \\
\text { ciones incorrectas. } \\
\text {-El documento con- } \\
\text { tiene muchos errores } \\
\text { de ortografía y gramá- } \\
\text { tica. } \\
\text {-El texto no sigue un } \\
\text { orden lógico y es ex- } \\
\text { tremadamente difícil } \\
\text { de entender. } \\
\text {-El diseño de fuente y } \\
\text { página no es acepta- } \\
\text { ble. } \\
\text {-Falta el título. } \\
\text { - No se incluye una } \\
\text { carátula de presenta- } \\
\text { ción (con el nombre, } \\
\text { el instructor y la sec- } \\
\text { ción de su clase). } \\
\text { 0 puntos }\end{array}$ \\
\hline
\end{tabular}




\section{Día 3 (tercera semana)}

El último día, los experimentos se concluyen procediendo a realizar la identificación de las semillas por medio del uso de lupas estereoscópicas. Aquí el alumnado abre las muestras y las divide en cuartos, un cuarto de la muestra se coloca en una caja de Petri la cual se divide en cuadrantes con algún marcador, para facilitar la separación de la muestra y proceden a la identificación de las mismas usando bibliografía de consulta (ver punto 4.3).
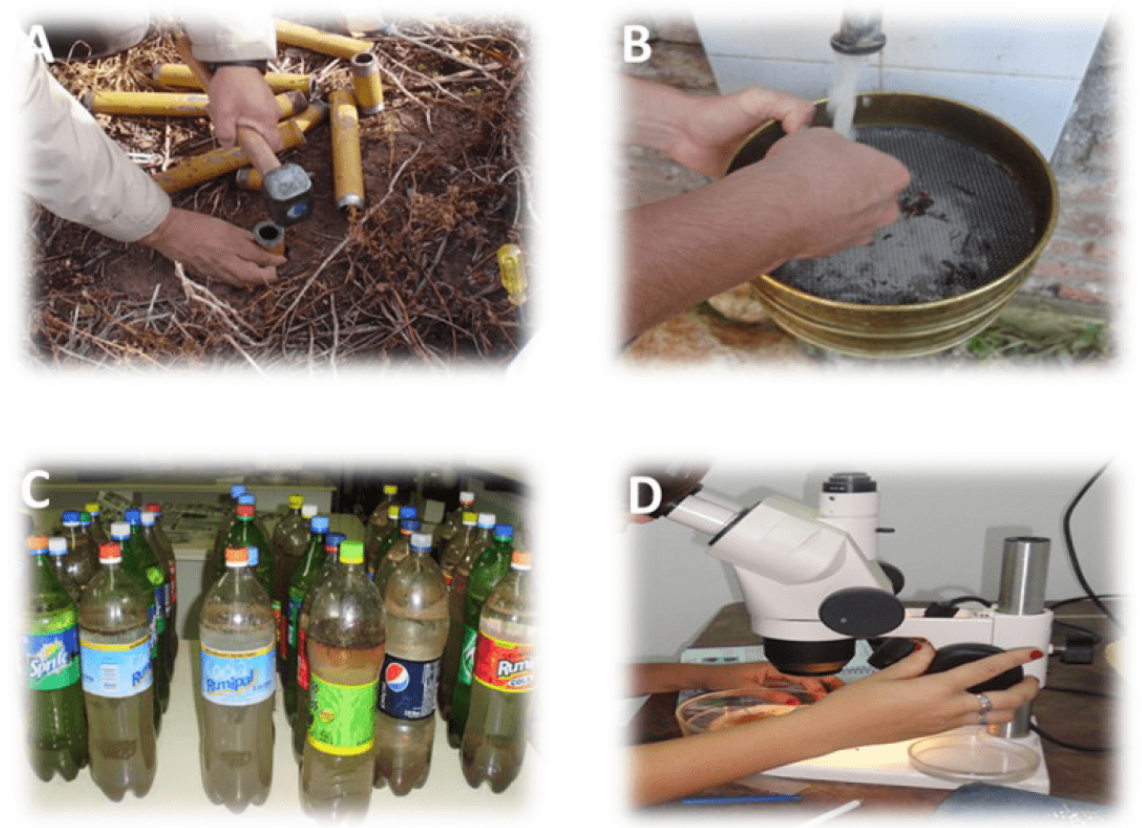

FIGURA 2

Imágenes del alumnado en los diferentes momentos de sus actividades. A) Tomando las muestras del BSS a campo mediante el uso de los cilindros. B) Usando los tamices para lavar las muestras del BSS. C) Botellas de gaseosa usada para la etapa de la dispersión de las muestras usando cloruro de sodio. D) Trabajo en la última etapa realizada en el laboratorio de semillas usando lupas estereoscópicas para la determinación de las especies presentes en las muestras del BSS

En la segunda etapa el alumnado comienza a organizar los datos obtenidos de los ensayos, basándose en análisis estadísticos (ver punto 4.4). El alumnado tendrá desde la finalización del practico 15 días más para concurrir a los laboratorios en horarios de extra clase o en clases de consulta para seguir analizando las especies y para la entrega del informe final. En el Anexo 1 se puede observar algunos resultados experimentales a los que arribaron los estudiantes en sus trabajos.

\section{EVALUACIÓN DE LOS APRENDIZAJES ADQUIRIDOS EN LA ACTIVIDAD PRÁCTICA}

El proceso de evaluación del alumnado es un proceso mucho más amplio que la mera aprobación o no de un cuestionario escrito o una exposición oral teórica. La evaluación es un proceso de delinear, obtener y proporcionar información útil para juzgar las alternativas de decisión. La evaluación es un proceso sistemático para determinar en qué medida el alumnado logra los objetivos educativos, por lo tanto, no puede ser tomada como fotografía de un momento en particular, sino que es un proceso continuo (Ibarra y Foresto, 2021). En nuestro caso tomamos en cuenta que la evaluación de los contenidos se llevó a cabo a lo largo de 
todos los momentos del trabajo prácticos (Tabla 1). La evaluación de esta clase de laboratorio programada se realiza durante los tres días de actividades prácticas y surge de la autoevaluación y autocrítica de los estudiantes durante su desarrollo. Además, como se mencionó anteriormente hacia el final de la actividad el alumnado realiza un informe escrito (tabla 1), en donde comunican los hallazgos de forma escrita. Para esto los estudiantes reciben una rubrica o guía para la realización del informe. Los aspectos que fueron tenidos en cuenta para la evaluación, como así también, los criterios de corrección se exponen en una rubrica (tabla 2) que muestra los parámetros cualitativos y cuantitativos que se tomaron en cuenta (Foresto, Nievas, Giordano y Bogino 2020).

Al finalizar la experiencia les solicitamos a nuestros estudiantes que nos brinden una devolución valorativareflexiva de esta actividad, para esto tuvieron la libertad de hacerla oral frente a sus compañeros o luego como un anexo a sus informes. Los comentarios escritos del alumnado encontraron en su mayoría a la actividad como muy motivadora y enriquecedora para aprender sobre la temática, mientras que los que observaron aspectos negativos en el mismo comentaron que se debía principalmente al tiempo y la dificultad involucrados en la redacción del informe. Algunos estudiantes sintieron que había demasiado que profundizar en la entrega del informe y que si bien no realizaron toda la actividad y cada uno trabajo un tratamiento y luego intercambiaron datos, hubieran preferido abarcar menos datos y poder trabajar con mayor profundidad un tratamiento particular. El alumnado destaco que la clase de discusión de resultados obtenidos para el informe fue muy importante, ya que les permitió profundizar en la temática y los ayudo en el proceso de escritura, incluso algunos y algunas estudiantes comentaron que el desarrollo de esta actividad fue muy importante durante el proceso de escritura de su proyecto de trabajo final de grado o tesina para poder optar al grado de Ingeniero Agrónomo. Algunos de los comentarios escritos positivos hechos por los estudiantes con respecto al ejercicio del banco de semillas fueron que permitió el estudio de tratamientos con diferentes tipos de labranzas, les hizo darse cuenta de cuántas semillas había en el suelo, y el impacto que tiene el BSS de malezas, en los posteriores manejos a desarrollar en el lote. En este sentido, les resulto interesante poner atención a la composición del BSS, ya que de esta forma, pudieron observar malezas con diferentes ciclos de vida y de crecimiento. Otra parte del alumnado disfruto mucho de los procedimientos metodológicos-experimentales de la actividad involucrados en el estudio de malezas, la separación de semillas y la posterior identificación de las mismas, como así también, la experiencia de trabajar en grupos.

El alumnado graduado de la carrera IA buscan empleo poco después, en campos relacionados en los sectores agronómicos. Los comentarios de nuestros exalumnos indican que las habilidades de laboratorio y las salidas al campo, favorecen sus capacidades de razonamiento, y esto mejora su competitividad en el mercado laboral, donde el campo de la sanidad vegetal es uno de los que genera más oportunidades laborales. Además, otros estudiantes que prefieren abrirse a un campo más académico sostienen que la elaboración de un informe de laboratorio simulaba la elaboración de un manuscrito científico con todos los apartados contenidos en un artículo estándar, y esto resulta muy beneficioso a la hora de la elaboración de su tesis posgrado, para quienes deciden continuar su formación de posgrado realizando alguna maestría o doctorado. De hecho, el trabajo práctico de laboratorio es uno de los aspectos clave del proceso de enseñanza y aprendizaje de las ciencias, por el aporte que puede hacer al alumnado en cuanto a habilidades adquiridas ya que son fundamentales para la realización de la investigación experimental (Caspers y Roberts-Kirchhoff 2003).

Desde el punto de vista del profesorado involucrado en la actividad, esta experiencia fue muy valiosa ya que proporciono una manera práctica, más cercana a lo que serán las futuras tareas que desarrollarán como IA, para aprender sobre la identificación de las semillas de las malezas, y el impacto en la biología y la ecología de las mismas en los sistemas agropecuarios. El alumnado tuvo la oportunidad de examinar las malezas en un sistema agrícola intervenido por diferentes tipos de labranzas y aprendieron un procedimiento simple de separación de semillas que les permitió conocer la composición y tamaño del BSS. El informe de laboratorio requería que los estudiantes integraran y aplicaran el material de la clase y resultó ser un buen ejercicio de redacción interpretativa y concisa. Lo que mas destaco el profesorado encargado de la actividad, fue que la 
mayoría de los informes estuvieron correctos y respondieron a los criterios de evaluación establecidos (tabla 2).

\section{CONSIDERACIONES FINALES}

El presente trabajo resulta un estudio exploratorio de una experiencia práctica vinculada BSS de malezas llevada a cabo por futuros IA. La propuesta empleo materiales de bajo costo y equipamiento que resulta de uso común en cualquier laboratorio universitario abocado al estudio de la biología y botánica de las plantas, la metodología es sencilla y fácilmente reproducible, como así también, libre de riesgos para la salud humana, siendo fácilmente aplicable en cualquier centro de enseñanza universitario o incluso secundario, si se minimizan la complejidad de algunas actividades. Este conjunto de experimentos permite al alumnado desarrollar diferentes habilidades como el manejo y diseño de ensayos experimentales; promover sus capacidades de discutir y resolver problemas prácticos; así como de sacar conclusiones de sus observaciones y los resultados obtenidos. Además, esta actividad práctica les pone en contacto con su futura carrera profesional y les brinda una posibilidad de iniciarse en el campo de la investigación. Esta propuesta intento aumentar la capacidad del alumnado para resolver problemas utilizando métodos científicos flexibles, desarrollar habilidades como el manejo de ensayos experimentales y la capacidad de discutir sus observaciones o resultados obtenidos.

Este programa de actividades proporcionó una alternativa a las actividades al aire libre con el propósito de adquirir conocimientos sobre la biología y la ecología de las malezas en los campos agrícola-ganaderos para alumnado universitario. Además, permitió a los estudiantes a integrar la teoría y la práctica para asegurar que comprendan mejor la relación entre ambos. Así, el desarrollo de este laboratorio práctico para el alumnado universitario ha sido diseñado para incentivarlos a incursionar en esta área incitando su curiosidad. Si bien la actividad requirió el acompañamiento de del equipo docente en la realización en las diferentes etapas, el alumnado pudo trabajar de manera grupal integrados con sus compañeros, favoreciendo la realización de actividades de manera colaborativa con sus pares.

Creemos que es importante crear un interés en el alumnado sobre temas que son novedosos y que actualmente son el foco de esfuerzos de investigación cada vez mayores, porque aumenta la comprensión de los estudiantes sobre la naturaleza del estado del arte de la investigación científica y, en consecuencia, puede llevar a un mayor entusiasmo en los estudiantes (Gasper y Gardner, 2013). En esta línea de pensamiento, resulta interesante pensar este trabajo como una actividad que pueda ser implementada en otros cursos, siguiendo la metodología propuesta en este trabajo, con la posibilidad de adaptarla según las necesidades específicas del alumnado al cual estará dirigida y, además, pueda servir como punto de partida para la elaboración de más estudios sobre las aplicaciones e implicancias educativas que pueden aportar los BSS para estudiantes de diversas edades y niveles educativos. En este sentido solo no resta decir que animamos al profesorado a desarrollar nuevos métodos de enseñanza para sus clases por medio de actividades que se realicen en el campo o laboratorios experimentales, motivándolos a salir del aula mediante una experiencia de aprendizaje integrada.

\section{Materiales SUPlementarios}

Anexo I (pdf) 


\section{REFERENCIAS}

Acosta, L., Agüero, R. (2001) El banco de propágulos de malezas en el agroecosistema: conocimiento actual y propuesta metodológica para su estudio. Agronomia Mesoamericana 12(2), 141-151.

Amuchástegui, M.A., Nuñez, C.O., Balzola, C., Zorza, E., Mulko, J. (2012) Distribución vertical de las semillas de malezas bajo diferentes tipos de labranzas en un sistema de rotación agrícola. XIV Jornadas Fitosanitarias Argentinas (compilado por Nora Raquel Andrada et al.), $1^{a}$ ed. Villa Mercedes. 10 pp. (cd-rom) ISBN 978-950-609-073-9.

Baskin, J., Baskin, C. (1985) The annual dormancy cycle in buried seeds: A continuum. Bioscience 3, 5, $492-498$.

Brewer, C. (2002) Conservation education partnerships in schoolyard laboratories: A call back to action. Conservation Biology 16 (3), 577-579.

Caspers, M. L., Roberts-Kirchhoff, E. S. (2003) An undergraduate biochemistry laboratory course with an emphasis on a research experience. Biochemistry and Molecular Biology Education 31, 303-307.

Di rienzo J.A., Casanoves, F., Balzarini, M.G., Gonzalez, L., Tablada, M., Robledo, C.W. (2018) InfoStat versión 2018. Grupo InfoStat, FCA, Universidad Nacional de Córdoba, Argentina. URL http://www.infostat.com.ar.

Ernst, R. D., Morici, E. (2013) Germinable seed bank of grasses in the caldenforests before and after dispersal differences. Revista de la Facultad de Agronomia de la Universidad Nacional de La Pampa 22(Suplemento 2 Tomo I), 39-44.

Foresto E. (2020) Aprendizajes formales, no formales e informales. Una revisión teórica holística. Contextos de Educación 29 (21), 24-36. Recuperado de: http://www2.hum.unrc.edu.ar/ojs/index.php/contextos/article/vie $\mathrm{w} / 1142 / 1234$.

Foresto, E., Amuchastegui M.A., Nuñez C.O., Zorza E. (2021) Comportamiento del banco de semilla de malezas en un sistema agrícola-ganadero intervenido por diferentes tipos de labranzas. Ciencia E Interculturalidad 28(01), 151-165. DOI: https://doi.org/10.5377/10.5377/rci.v28i01.11466

Foresto, E., Martin R.B. (2020) Acercamientos a la conceptualización de la botánica. Un estudio con ingresantes de ingeniería agronómica. Bio-grafía, 13(25). DOI: https://doi.org/10.17227/bio-grafia.vol.13.num25-12322.

Foresto, E., Nievas, F., Giordano, W., Bogino, P. (2020) Novel, simple and inexpensive programmed lab experiments to evaluate the biocontrol activity of rhizobacteria on fungal phytopathogens. Journal of Biological Education 1-17. DOI: $10.1080 / 00219266.2020 .1858934$.

Gasper, B.J., Gardner, S.M. (2013) Engaging students in authentic microbiology research in an introductory biology laboratory course is correlated with gains in student understanding of the nature of authentic research and critical thinking. Journal of microbiology \& biology education 14, 25-34.

Ibarra, L. E., Foresto, E. (2021). An experimental approach to evaluate osmosis and tonicity on white blood cells by flow cytometry for biomedical physiology students. Journal of Biological Education 1-14. DOI: https://doi.org /10.1080/00219266.2021.1941188

Ju, E. J., Kim, J. G. (2011). Using soil seed banks for ecological education in primary school. Journal of Biological Education 45(2), 93-101.

Ju, E. J., Kim, J. G. (2012). Development of Educational Program using Soil Seed Bank for Promoting Ecological Literacy. Journal of Korean Elementary Science Education 31(3), 284-297.

Leck, M. A., Parker V. T., Simpson R.L. (1989) Ecology of soil seed banks. San Diego, CA: Academic Press.

Leguizamón, E. S., Roberts, H. A. (1982) Seed production by an arable weed community. Weed Research 22(1), 35-39.

Mayoral, O. (2019) Las plantas como recursos didácticos. La Botánica en la enseñanza de las Ciencias. Flora Montiberica 73, 93-99.

Miles, J. (1979) Vegetation dynamics. London: Chapman and Hall.

Pascoe, F. (1994) Using soil seed banks to bring plant communities into the classroom. The American Biology Teacher 56(7), 429-432. 
Emiliano Foresto, et al. El banco de SEmillas del Suelo. Una metodología experimental SENCiLla, RE...

Pucheta, E., Cabido, M., Díaz, S., Funes, G. (1998) Floristic composition, biomass, and aboveground net plant production in grazed and protected sites in a mountain grassland of central Argentina. Acta Oecologica 19(2), 97-105.

Regnier, E. E. (1995). Teaching seed bank ecology in an undergraduate laboratory exercise. Weed technology 9(1), 5-16.

Sabattini, R. A., Sione, S. M., Ledesma, S. G., Rosenberger, L. J., Wilson, M. G., Loker, M. F., Cinto, M. (2018) Banco de semillas de especies arbóreas en bosques nativos del espinal (Entre Ríos). Ciencia, Docencia y Tecnología 8(8) 55-90.

Simmons, D. (1998) Using natural settings for environmental education: Perceived benefits and barriers. The Journal of Environmental Education 29 (3), 23-31.

Sione, S. M. J., Ledesma, S. G., Rosenberger, L. G., Galliussi, R., Sabattini, R. A. (2015) Soil seed bank in two successional stages of the native forest in Entre Ríos. Quebracho 23, $62-76$.

Tilling, S. (2004) Fieldwork in UK secondary schools: Influences and provision. Journal of Biological Education 38, 54-58.

\section{INFORMACIÓN ADICIONAL}

Para citar este artículo: Foresto. E., Amuchástegui, M.A., Nuñez, C. O. y Ibarra, L. E. (2022) El banco de semillas del suelo. Una metodología experimental sencilla, reproducible y de bajo costo para aprender sobre la biología de las malezas. Revista Eureka sobre Enseñanza y Divulgación de las Ciencias 19(1), 1203. doi: 10.25267/Rev_Eureka_ensen_divulg_cienc.2022.v19.11.1203 